\title{
Dispositif d'alerte et de déclaration des maladies infectieuses
}

\section{Systems of early warning and reporting of infectious diseases}

\author{
D. Bitar - C. Saura \\ (C) SRLF et Springer-Verlag France 2011
}

Au-delà de l'urgence à soigner un patient, le réanimateur est, comme tout praticien hospitalier, un maillon essentiel du dispositif de surveillance et de lutte contre les maladies infectieuses. Avec le signalement des infections liées aux soins, il contribue à la maîtrise des risques nosocomiaux et à l'amélioration des pratiques. Devant une infection communautaire grave, par exemple une infection invasive à méningocoque, il est amené à organiser la prophylaxie de l'entourage familial. À travers le signalement des maladies à déclaration obligatoire (DO), il contribue à réduire la diffusion de l'infection : à la réception du signalement, l'agence régionale de santé (ARS) organise la chimioprophylaxie et, le cas échéant, la vaccination dans l'entourage familial, l'école, la crèche, le lieu de travail, etc. Le signalement permet en outre à l'ARS de détecter des cas groupés lorsque d'autres cas sont signalés à partir d'autres services ou d'autres hôpitaux, déclenchant alors une alerte et une investigation spécifique. Dans un second temps, les données de la DO sont regroupées et analysées par l'Institut de veille sanitaire (InVS) afin d'étudier les caractéristiques des patients et des souches circulantes, d'analyser les tendances et de proposer au besoin une adaptation de la stratégie de prévention et de lutte.

Certains articles publiés dans ce numéro font référence aux maladies à DO (rougeole, chikungunya), d'autres concernent des infections faisant l'objet d'une surveillance spécifique comme la grippe. Ces pathologies illustrent la richesse et la complexité des dispositifs de surveillance actuels, mis en place afin de contrôler la diffusion des infections et s'adressant à des pathogènes connus ou à des phénomènes nouveaux. L'organisation intrinsèque et les partenaires de ces systèmes peuvent varier, mais les grandes lignes du dispositif restent les mêmes (Fig. 1) : la surveillance spécifique des maladies infectieuses est basée le plus souvent sur l'envoi d'informations à un premier niveau (local ou régional) chargé de l'analyse et de l'intervention

D. Bitar $(\bowtie) \cdot$ C. Saura

Département des maladies infectieuses,

Institut de veille sanitaire, 12, rue du Val-d'osne,

F-94415 Saint-Maurice cedex, France

e-mail : d.bitar@invs.sante.fr immédiate. Pour les infections nosocomiales, il s'agit du Comité de lutte contre les infections nosocomiales (CLIN), puis du Centre de coordination de la lutte contre les infections nosocomiales (CClin) et de l'ARS. Pour les maladies à DO, le signalement et la fiche de déclaration sont adressés aux ARS (plateforme régionale de veille sanitaire ou leurs délégations territoriales) qui ont remplacé les anciennes directions départementales des affaires sanitaires et sociales (Ddass). Enfin, l'InVS, avec les cellules interrégionales d'épidémiologie (Cire) qui sont ses cellules en région, organise, impulse et coordonne les systèmes de surveillance, et analyse les données récoltées au niveau régional et national.

Pour compléter les systèmes de surveillance spécifique des maladies infectieuses connues, et dans un objectif d'anticipation et de détection précoce de maladies infectieuses émergentes, un dispositif de signalement des événements inhabituels susceptibles de représenter une menace pour la santé publique est prévu. Ces événements peuvent inclure une pathologie avec une expression clinique ou une gravité inhabituelle, ou une souche présentant un profil de résistance rare ou nouveau. Les équipes de réanimation sont en première ligne pour identifier de tels phénomènes. Suite au signalement à l'ARS et après une étape de vérification des informations collectées, l'analyse du risque pour la santé publique est organisée de manière multidisciplinaire, en faisant appel à l'expertise de la Cire et de l'InVS pour l'investigation du phénomène et la recherche d'événements similaires dans d'autres régions françaises ou dans le monde. Cette analyse mobilise également l'expertise des cliniciens et biologistes hospitaliers concernés, celles des centres nationaux de référence et d'autres disciplines potentiellement concernées (santé animale, environnementale, etc.). L'alerte de santé publique de portée locale, régionale ou nationale, est ensuite déclenchée sur la base de cette analyse.

Après la phase d'identification du phénomène émergent et d'analyse $\mathrm{du}$ risque qu'il représente pour la santé publique, des systèmes de surveillance sont mis en place par l'InVS et les Cires pour suivre l'impact du phénomène. Ainsi, lors de la pandémie grippale en 2009, dans une première phase, l'ensemble des cas potentiellement infectieux (exposés dans des pays où le virus circulait) devaient 


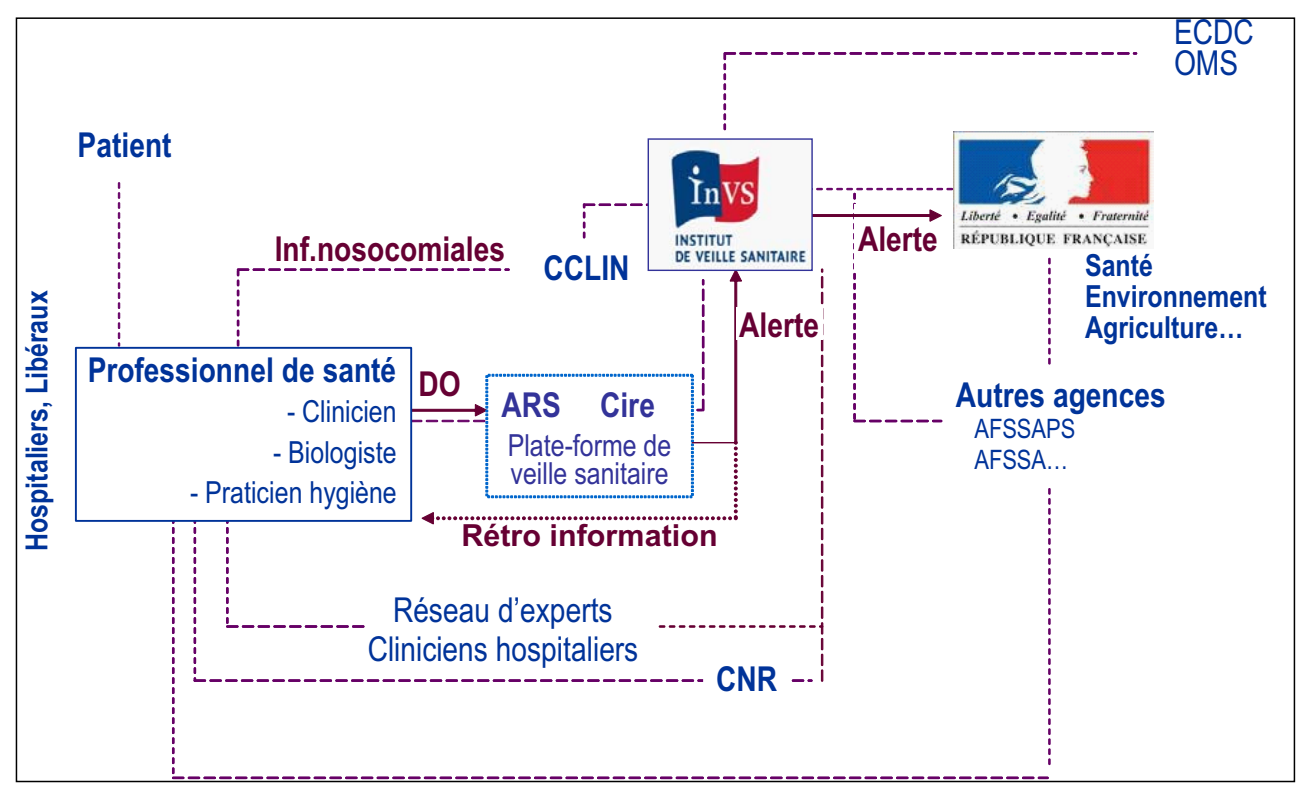

Fig. 1 Schéma simplifié de l'organisation du dispositif d'alerte et de déclaration des maladies infectieuses en France

être identifiés par les Centres 15 et signalés à l'InVS. La surveillance a ensuite été restreinte aux cas groupés survenant sur le territoire national, puis exclusivement aux cas hospitalisés, puis enfin aux cas les plus graves admis en réanimation. La participation des réanimateurs à la surveillance des cas graves de grippe a ainsi permis d'améliorer les connaissances sur l'infection, les groupes à risque de formes graves, l'intérêt du traitement, les cibles prioritaires de la vaccination, etc. De tels ajustements dans la surveillance nécessitent d'informer régulièrement les partenaires chargés du recueil de données et pour l'InVS de développer des partenariats à l'échelon régional et national.

C'est dans cet objectif qu'un groupe de réflexion a été mis en place en 2010, associant l'InVS, les Cires et des réanimateurs de la SRLF, de la SFAR et du GFRUP, afin de construire ce partenariat « à froid » et de renforcer les interactions entre professionnels de la réanimation et de la veille sani- taire. Dans le contexte évolutif de l'organisation de la veille sanitaire à l'échelon régional, il importe que le praticien reçoive une information aussi claire et pratique que possible sur les circuits de signalement compte tenu des nombreuses surveillances et vigilances auxquelles les réanimateurs contribuent activement (hémo- et pharmacovigilance, infections liées aux soins, DO, etc.). Dans le même temps, il convient de renforcer la rétro-information auprès du praticien pour donner de la visibilité sur l'utilité de sa contribution et lui apporter des informations sur les alertes en cours potentiellement utiles à sa pratique. Les Cires, en tant que cellules de l'InVS en région en lien direct avec les ARS, ont un rôle important d'animation des réseaux pour améliorer la connaissance de nos rôles et contributions respectifs dans la veille et la surveillance des maladies infectieuses.

Pour plus d'information : www.invs.sante.fr 